\title{
Influence of duty cycle and off time of comparison-tone pulse trains on the measurement of perstimulatory loudness adaptation
}

\author{
W. H. AHAUS, T. E. STOKINGER, and MARGARET A. WYLDE \\ Veterans Administration Hospital and University of Oklahoma College of Health \\ Oklahoma City, Oklahoma 73104
}

\begin{abstract}
An experiment was conducted to determine if duty cycle and off time of tone pulses presented to the comparison ear influence adaptation measured at the opposite (test) ear. Eight subjects were adapted for $5 \mathrm{~min}$ to a $1-\mathrm{kHz}$ pure tone at $60 \mathrm{~dB}$ SPL. Using a tracking procedure, adaptation was measured under five comparison-signal conditions, each comprised of 1-kHz pulse trains having different on/off times. The on/off times (in milliseconds) were: $200 / 800$ (20\% duty cycle); 500/500, 200/200, and 800/800 (50\% duty cycle); and $800 / 200$ ( $80 \%$ duty cycle). Adaptation was found to increase as the duty cycle of the comparison tones increased from $20 \%$ to $80 \%$. This was evident even when attempts were made to account for the extent to which pulse trains are perceived as softer than continuous signals at the same level (the so-called LOT effect). For the $50 \%$-duty-cycle conditions, similar amounts of adaptation were measured whether the on/off times of the signals were 200,500 , or 800 msec.
\end{abstract}

Adaptation in the peripheral auditory system was demonstrated physiologically as early as 1935 by Derbyshire and Davis, who showed a marked reduction in the amplitude of the auditory nerve action potential during prolonged suprathreshold stimulation by pure tones.

Auditory adaptation can be demonstrated psichophysically as well, at least under certain listening conditions. It has been referred to in the literature as perstimulatory loudness adaptation and has been detined as the decrease in the loudness of an ongoing auditory stimulus. Adaptation was a popular topic in psychoacoustic research during the 1950 s and early 1960s.

The traditional approach to measuring adaptation behaviorally involves presenting the adapting signal to one ear and a variable-intensity comparison signal of the same frequency to the other. The magnitude of the adaptation produced in the adapting ear is then detined as the amount. over time, by which the intensity of the comparison signal in the nonadapting ear must be decreased in order for it to be judged equal in loudness to the adapting signal. Using such a procedure. many investigators have reported adaptation values ranging from 20 to $50 \mathrm{~dB}$ over a 3-7-min tine period (see Small. 1963). It is important to note that measuring adaptation in this way necessarily involves stimulating the nonadapting ear at rarious times during the adapting period, resulting in at least some binaural interaction.

In the past few years, a number of additional adaptation studies have appeared in which the

This research was supported by funds from the Research and Education Committee. Veterans Administration Hospital. Oklahoma City. Oklahoma. under Project 20-69. experimenters have attempted to eliminate these binaural cues, either (1) by stimulating the ear monaurally and asking a listener either to adjust the intensity of the tone to maintain a constant loudness or to judge periodically whether or not its loudness had changed (Mirabella. Taub. \& Teichner, 1967; Wiley, Small. \& Lilly, 1973); (2) by using a delayed balance procedure. where the comparison signal is presented to the nonadapting ear after termination of the adapting tone (Petty. Fraser, \& Elliott, 1970; Stokinger, Cooper, Meissner, \& Jones, 1972; Stokinger \& Studebaker. 1968); or (3) by employing heterophonic balance conditions, whereby the adapting and comparison ears receive signals that differ substantially in frequency (Bray, Dirks, \& Morgan. 1973; Fraser. Petty, \& Elliott. 1970; Morgan d Dirks. 1973).

The basic result of all these experiments was the same: when binaural interaction was eliminated. little or no adaptation occurred. at least in normal-hearing listeners. This finding obtained despite the fact that we have known for 40 years that afferent eighth-nerve fibers do adapt to monotic stimulation! Thus, perstimulatory adaptation is now considered by modern reviewers (Ward. 1973) to be a centrally mediated phenomenon, or, if partly peripheral. at least influenced by efferent action resulting from simultaneous contralateral stimulation.

Given, then, that an interaction between the adapting and comparison ears is a requisite for producing adaptation in normals, our research interests have been directed toward a closer investigation of the nature of these binaural influences. It seems reasonable to wonder, for example. whether varying amounts of binaural 
interaction might produce varying magnitudes of adaptation.

Stokinger, Cooper, and Meissner (1972a) investigated this hypothesis and measured adaptation for different comparison-signal conditions. Using a single, simultaneous dichotic loudness balance (singleSDLB) paradign, they found significantly more adaptation to an $8-\mathrm{sec}, 1-\mathrm{kHz}$ tone when a contralateral comparison tone was $2,000 \mathrm{msec}$ in duration than when its duration was only $200 \mathrm{msec}$. In addition, when their listeners were adapted for $5 \mathrm{~min}$ to a $1-\mathrm{kHz}$ tone and a multiple-SDLB paradigm was employed, more adaptation resulted when the $1-\mathrm{kHz}$ comparison tones had a duty cycle of $50 \%$ ( 1 sec ON. $1 \mathrm{sec}$ OFF) than when the duty cycle was only $16-2 / 3 \%$ ( 1 sec ON, 5 sec OFF).

The relation between adaptation and duty cycle of the comparison signal may not be so straightforward, however. In a different paper, Stokinger, Cooper, and Jones (1971) found no signiticant difference in the amount of adaptation measured when comparisontone duty cycles were $33-1 / 3 \%$ and $66-2 / 3 \%$. Thus, it could be that there are limits beyond which increased contralateral stimulation no longer influences the magnitude of adaptation. A further investigation of the effects of varying amounts of contralateral stimulation on adaptation seemed to be in order.

In the experiments of Stokinger, Cooper, and Jones (1971) and Stokinger, Cooper, and Meissner (1972a), the multiple-SDLB method was implemented with a double-staircase procedure (Cornsweet, 1962) for adjusting the comparison-signal intensity level. The double-staircase procedure, however, fixes the attenuation step size and thereby limits the capacity of the attenuation mechanism to follow the changes in the loudness of the adapting signal. Too large a step size decreases the precision with which one may measure adaptation. Too small a step size prohibits the attenuation mechanism from matching and recording the rapid loudness changes which occur at the start of the adapting period. To eliminate this problem. comparison-signal attenuation in this experiment was adjusted by a tracking method with an attenuation rate of $4 \mathrm{~dB} / \mathrm{sec}$. The tracking method does, however, contribute another problem to the measurement of adaptation. That problem is the LOT effect.

When a listener is required to track equal loudness using trains of short-duration tone bursts as comparison signals, his perception almost surely will be influenced by the so-called LOT (for lengthened off time) effect (Hattler, 1968). The phenomenon refers to the fact that when pulse trains are presented at a suprathreshold level, they are judged to be less loud than a continuous signal at the same frequency and intensity, even though the duration of the individual bursts exceeds the critical duration necessary for complete temporal integration of loudness (Zwislocki,
1969). Apparently, the loudness of the train as a whole is perceived by the auditory system, and not the loudness of each individual tone pulse. The LOT effect for a particular pulsed tone is the difference (in decibels) between the loudness of the train of tone pulses and the loudness of a steady tone at the same intensity level. The size of the effect has been shown to vary with both the duty cycle and the off time of the signals (Hattler, 1968).

In the usual measurement paradigm, adaptation is detined as the amount by which the intensity of the comparison signal must be decreased to maintain equal interaural loudness. The LOT effect would act in the opposite direction of adaptation. That is, the comparison tone would not sound louder than the adapting tone as quickly as it would if the LOT effect were not acting. since the comparison tones would have been perceived initially (at the time the adapting tone is made continuous) as softer than the adapting signal.

In fact, for the signal conditions that produce a large LOT effect, subjects often increase the level of the comparison tone initially, implying an increase in loudness of the adapting signal, whereas, in reality, this results from a reduced loudness of the pulsed comparison signal in relation to the continuous adapting signal (Morgan \& Dirks, 1973; Stokinger, Cooper, \& Meissner, 1972b).

Because varying the duty cycle of the comparison-tone pulses would likely produce various-sized LOT effects, the influence of the effect on adaptation would not be constant, but would vary with the different experimental conditions. An attempt was made, therefore, to neutralize the LOT effect in our data by measuring the size of each listener's LOT effect for each experimental condition, and correcting that listener's adaptation data by the amount of his measured LOT effect.

The purpose of this experiment, then, was to investigate the effect of varying the duty cycle and period of comparison-tone pulses on adaptation, both when the LOT effect is disregarded and when a correction for it is taken into account.

\section{METHOD AND PROCEDURE}

Eight practiced listeners. four females and four males between the ages of 22 and 32 years. served as subjects in the experiment. for which they were paid a nominal hourly wage. All subjects had clinically normal hearing bilaterally, and none displayed more than a 5 -dB difference in threshold at $1 \mathrm{kHz}$ between their right and left ears.

Listeners were adapted for 5 min to a continuous $1-k H z$ pure tone at $60 \mathrm{dBSPL}$. Adaptation was measured under five comparison-signal conditions. The five comparison signals were $1-\mathrm{kHz}$ tone bursts having either the same period but different duty cycles or the same duty cycle but different periods. The switching envelopes for the pulsed tones are illustrated in Figure 1. Three of the comparison tones were pulsed with a $50 \%$ duty cycle, with on/off times of 200,500 , and $800 \mathrm{msec}$. The remaining two signals had 1 -sec periods, as did the second of the three $50 \%$-duty-cycle 
signals. The three signals with 1 -sec periods differed in their duty cycles. one having a $50 \%$ duty cycle, another having an on time of $2(0)$ msec and an off time of $800 \mathrm{msec}(20 \%$ duty cycle), and the final signal being on $800 \mathrm{msec}$ and off $200 \mathrm{msec}(80 \%$ duty cycle). All signals had a $10-\mathrm{msec}$ rise-decay time.

Both the adapting and comparison signals were generated by the same audio oscillator. its output simply being split and routed through appropriate switching and attenuator networks to the two earphones. The pulsed comparison signals passed through a phase inverter inserted just before the earphone, so that for all conditions a 180-deg phase difference existed between the adapting and comparison signals. All subjects received the adapting signals in their right ears, while the comparison tones were always delivered to their left ears.

Data were collected using a Békésy tracking method, with the subject having control over the level of the comparison tones by means of a recording attenuator with a pen speed of $4 \mathrm{~dB} / \mathrm{sec}$.

Previous experience measuring adaptation in this laboratory has shown us that there is considerable variability not only between but also within subjects. In an effort to reduce the effect of this variability, subjects received each of the tive comparison-signal conditions 10 times, in quasi-random order. An individual listener's data for a particular signal condition were taken as the mean of those 10 trials.

Each listening session for the various experimental conditions comprised three parts: deternination of the subject's LOT effect for that particular condition. determination of the subject's preadapting balance level, and determination of balance levels throughout the $5 \mathrm{~min}$ of continuous stimulation of the adapting ear. The specific paradigm employed is shown in Figure 2.

One purpose of the investigation was to determine the extent to which the LOT effect might have an influence on adaptation measured when trains of tone pulses are used as comparison stimuli. An effort was made to determine the magnitude of each listener's LOT effect for each experimental condition during the first part of each listening session. Duplicating the procedure employed by Hattler (1968), the tone pulses were first presented at $60 \mathrm{~dB} \mathrm{SPL}$ to the subject's left (comparison) ear for $30 \mathrm{sec}$. This, according to Hattler. insures that if the subject adapts to the pulsed tone. the ad aptation will have had time to asymptote. At the end of this 30-sec period. the tone was made continuous and presented for $5 \mathrm{sec}$. Following the $5-\mathrm{sec}$ presentation at $60 \mathrm{~dB}$ SPL. the signal was again made pulsed, but the subject was now given control over its level via the recording attenuator. The subject was instructed to keep the loudness of the pulsed tone equal to the remembered loudness of the 5-sec steady signal.

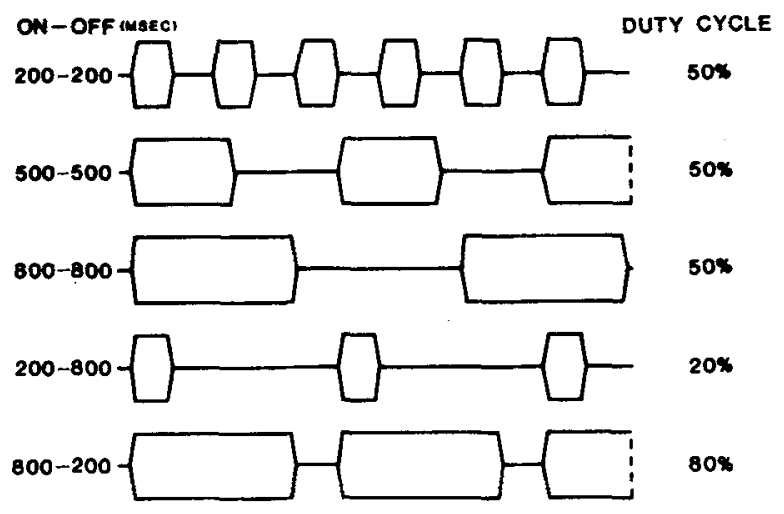

Figure 1. Switching envelopes of the 1-kHz comparison-tone pulse trains. The upper three signals were pulsed with a $50 \%$ duty cycle and had periods of $0.4,1.0$, and 1.6 sec. The hower two signals had 1 -sec periods and duty cycles of $20 \%$ and $80 \%$. Thus, three signals had the same duty cycle but different periods, and three had equal periods but different duty cycles.

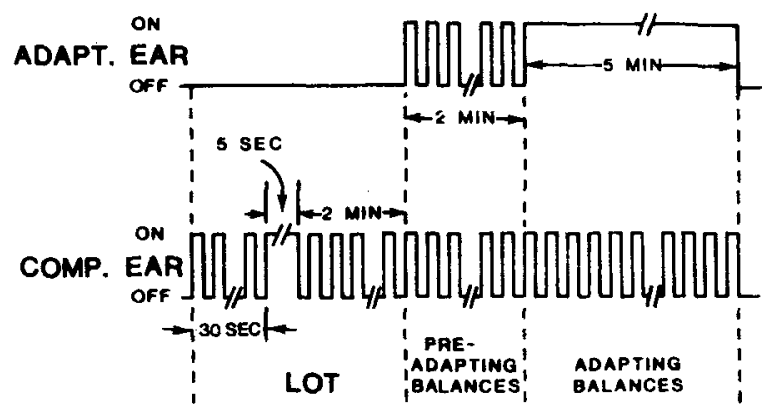

Figure 2. Schematic representation of the experimental paradigm. Listening sessions comprised three parts: (1) determination of the LOT effect, the phenomenon whereby the perceived loudness of a pulse train is less than the loudness of an equally intense continuous signal (see text); (2) determination of the preadapting balance level; and (3) determination of balance levels during the 5 -min adapting period.

This remembered-loudness percept was tracked for 2 min. Tracking levels were recorded at successive $20-\mathrm{sec}$ intervals throughout the 2 -min period, and these six measurements were averaged. This average tracking level, minus $60 \mathrm{~dB}$ (the level of the steady. 5-sec signal), was taken as the LOT effect for that particular subject for that condition on that particular day. For example, if a subject's average tracking level were $64.6 \mathrm{~dB}$, he is saying that, in order for this train of tone pulses to sound equal in loudness to the steady 60 -dB-SPL reference tone. they must be at a level of $64.6 \mathrm{~dB}$. The LOT effect, therefore, would be $4.6 \mathrm{~dB}$ and would represent the extent to which the pulsed signals were perceived as being less loud than a steady signal of equal amplitude.

At the end of the 2-min LOT tracking period, the pulsed tones were introduced simultaneously into the adapting ear, marking the beginning of the preadapting balance period. Under this condition, listeners perceive a tonal image that varies in its position within the head depending on the interaural intensity difference. This is true in spite of the fact that the signals were $180 \mathrm{deg}$ out of phase. The subjects were instructed to keep this image located at the midline by varying the level of the signal in the comparison ear. The subject made these preadapting balances for $2 \mathrm{~min}$. The preadapting level was taken as the mean of the balance levels determined at six successive 20 -sec intervals within the $2 \mathrm{~min}$.

At the end of the preadapting period, the tone in the adapting ear was made continuous, and the adapting period was begun without delay. Since the two ears then received different signals (a pulsed tone to one ear, a steady tone to the other), actual interaural loudness differences could be perceived, so that the subject was no longer asked to make lateralization judgments. During the adapting period, the subjects were instructed to keep the tone pulses in the comparison ear equal in loudness to the steady signal in the adapting ear. Balance levels were recorded at 20 -sec intervals throughout the 5 -min adapting period.

\section{RESULTS AND DISCUSSION}

Standard deviations were computed at the 300 -sec time point (maximal adaptation) for each subject for each of the five comparison signals to get some idea of listener variability over the 10 repetitions. Standard deviations for the eight subjects ranged from $3.92 \mathrm{~dB}$ to $12.33 \mathrm{~dB}$, with a mean value of $6.52 \mathrm{~dB}$.

The group data are represented in Figures 3 and 4. The comparison-tone sound pressure level required to 


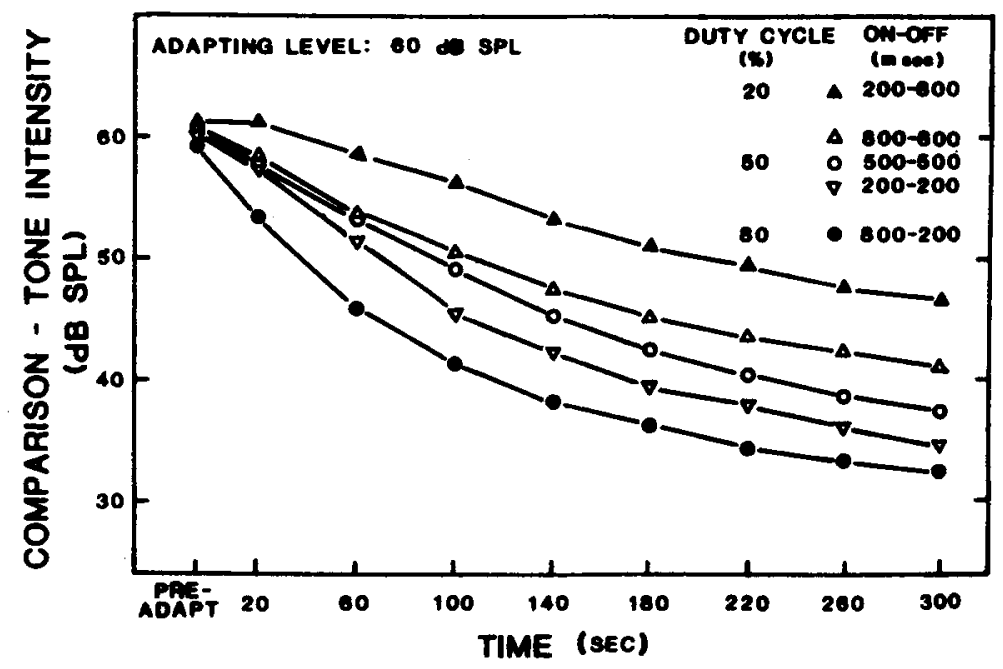

Figure 3. Group data for the five signal conditions, not corrected for the LOT effect. Comparison-tone sound pressure levels are plotted at 40 -sec intervals throughout the 5-min adapting period. Each point is the mean of $\mathbf{8 0}$ observations.

maintain equal loudness to the 60-dB adapting signal is shown in Figure 3, plotted as a function of time along the abscissa. These data are not corrected for the LOT effect. Although balance-level data were recorded every $20 \mathrm{sec}$, they are shown only at $40-\mathrm{sec}$ intervals for purposes of clarity. Each datum point represents the mean of 80 measurements (10 observations for each of the eight subjects).

It can be seen from Figure 3 that the magnitude of the adaptation clearly depends upon the temporal characteristics of the comparison tone. As duty cycle is increased from $20 \%$ to $80 \%$, adaptation also increases. For tones pulsed with a $50 \%$ duty cycle, adaptation increases slightly as the off time of the comparison tones is decreased. The average maximum adaptation ranged fron about $14 \mathrm{~dB}$ for a comparison tone pulsed at a $20 \%$ duty cycle to about $28 \mathrm{~dB}$ for an $80 \%$-duty-cycle comparison signal. The rate of growth of adaptation with time also increased with comparison-tone duty cycle, at least during about the first 2 min of stimulation.

It is impossible to be sure whether adaptation had reached asymptote for any duty-cycle condition, although the slight curving of the functions after about $2 \mathrm{~min}$, especially for the highest duty cycle, suggests that it may have at least been close. Similar adaptation magnitudes and time courses have been reported by others using a simultaneous loudness balance technique (Small, 1963).

An attempt was made to determine the extent to which these results may have been influenced by the LOT effect. Table 1 shows the mean LOT effect values for the five comparison signals as well as the standard deviations. As previously discussed, the LOT effect is the extent to which pulse trains are perceived as being less loud than a continuous signal of the same level. The larger the size of the LOT effect, the more the loud ness is reduced by the signal's being pulsed.
It can be seen that the magnitude of the effect varies with signal off time; it is smallest for the $20-\mathrm{msec}$ off time and increases with increasing off time, reaching a maximum of $5.4 \mathrm{~dB}$ for the $200 \mathrm{msec}$ ON, $800 \mathrm{msec}$ OFF condition. The LOT effect for each of the five comparison signals was applied to the group data.

These corrected group data are displayed in Figure 4 . The coordinates and parameters are identical to those in Figure 3, but the LOT effect has simply been added to the amount of adaptation under each comparison signal condition. For example, the comparison signal SPL for the 20\%-duty-cycle condition (filled triangles) was lowered (implying more adaptation) at every point by $5.4 \mathrm{~dB}$. Similarly, the function for the $80 \%$-duty-cycle condition was shifted downward $1.2 \mathrm{~dB}$. The preadapting balance level was, of course, left unchanged, because for that portion of the measurement procedure listeners received pulsed tones to both ears rather than pulsed tones to one ear and a continuous tone to the other.

From Figure 4, it is still evident that adaptation increases systematically with increasing comparisontone duty cycle. For the $50 \%$-duty-cycle signals, however, correcting for the LOT effect brings together the functions for the three off times.

Table 1

Means and Standard Deviations of the LOT (Lengthened Off Time) Effect for the Five Experimental Conditions, Averaged Across the Eight Listeners

\begin{tabular}{lccccc}
\hline $\begin{array}{l}\text { On/Off } \\
\text { (msec) }\end{array}$ & $200 / 200$ & $800 / 200$ & $500 / 500$ & $800 / 800$ & $200 / 800$ \\
\hline Mean & 1.2 & 1.2 & 4.0 & 4.9 & 5.4 \\
SD & 1.4 & 1.1 & 1.7 & 2.4 & 1.6 \\
\hline
\end{tabular}

Note-LOT-effect values are the amount (in decibels) by which the average remembered-loudness tracking level for a particular condition exceeded the loudness of the 60-dB-SPL reference signal. 
Figure 4. Group data for the five signal conditions, corrected for the LOT effect. Comparison-tone sound pressure levels are plotted at 40 -sec intervals throughout the 5 -min adapting period. Each point is the mean of 80 observations.

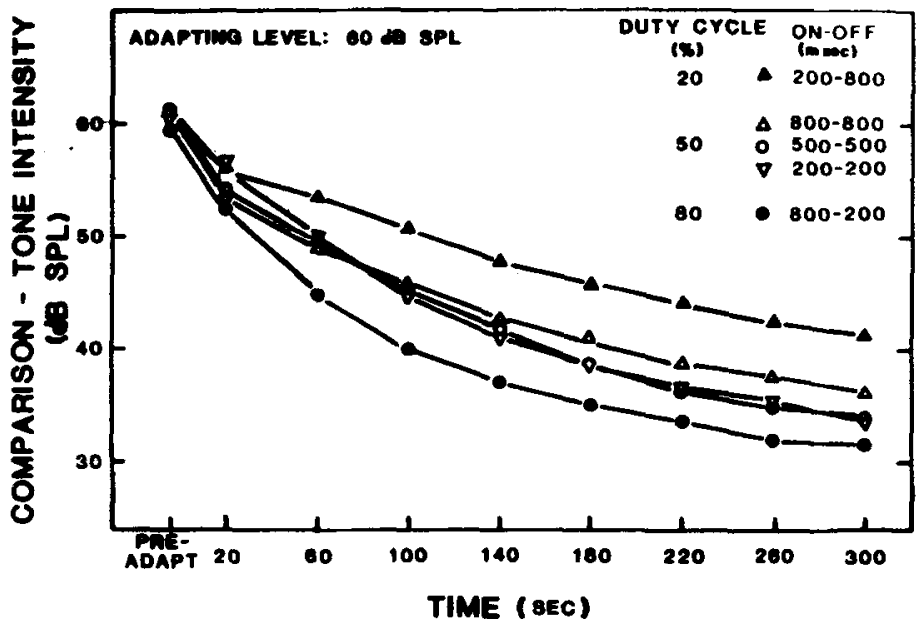

The trends in the data are similar for all eight listeners. Data for three of those eight subjects, one who displayed slight, one who displayed moderate, and a third who exhibited a large amount of adaptation, are plotted in Figure 5 to illustrate this similarity. Each subject's data for the three duty

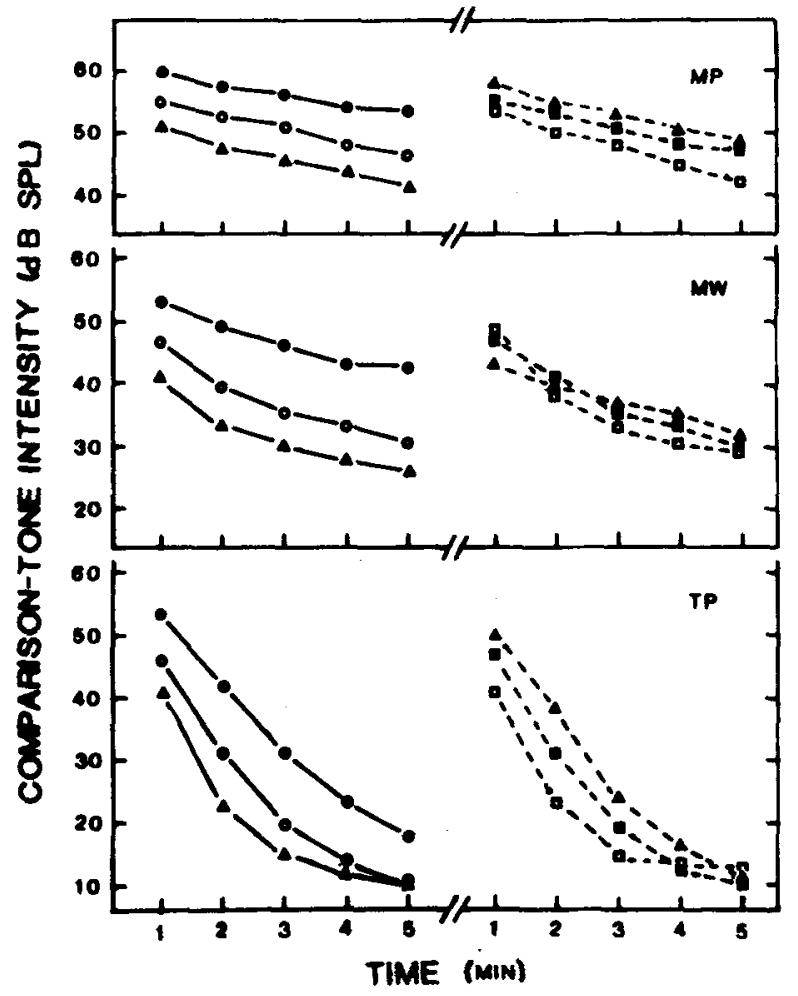

Figure 5. Representative individual data for three of the eight subjects, corrected for the LOT effect. The left-hand portion shows adaptation for the $20 \%(-0), 50 \%(\mathrm{O}-\mathrm{O}$ ), and $80 \%(\triangle-\infty)$ duty cycles. The right-hand portion displays adaptation for a $50 \%$-duty-cycle signal and periods of 0.4 (口- $-\square), 1.0(\square-\ldots)$, and $1.6(\Delta-\ldots, \Delta)$ sec. cycles are shown in the left-hand portion of the graph, and the functions for the $50 \%$-duty-cycle condition for the three off times or periods are displayed in the right-hand portion. Although the extent of the adaptation as well as the shapes of the individual functions are different, the subjects all show the same tendency for adaptation to increase with duty cycle and for similar amounts of adaptation to result from the $50 \%$-duty-cycle condition for the three on/off times.

We conclude that increasing binaural interaction by increasing the percentage of time the comparison tone is actually on results in increased adaptation; varying the off time of the pulses for a particular duty cycle (at least for $50 \%$ ) does not substantially affect the adaptation measured.

On the one hand these results are quite consistent with a conceptual model of adaptation that depends on binaural interaction. In another sense, however, some questions arise. One might theorize, for example, that as the duty cycle of the comparison tone increases, the comparison ear itself might adapt and thereby reduce the apparent adaptation occurring in the adapting ear. Conceivably, a point (duty cycle) might be reached, where adaptation in the comparison ear comes so close to counteracting that in the adapting ear that the experimental results would show a decrease in adaptation with increasing comparisontone duty cycle above that point.

Clearly, that did not occur, at least for the conditions we employed. It could well be that the comparison ear did adapt; but if so, there was enough of a concomitant increase in test-ear adaptation, due to increased binaural interaction, to more than offset any comparison-ear adaptation effect.

These results further support the notion that, not only is binaural interaction necessary to measure adaptation, but the extent of this interaction directly influences the amount of adaptation measured. Our data show this to be true for comparison signals 
comprising trains of short pulses, while other data from our laboratory (Stokinger et al., 1972a) have demonstrated this finding for single comparison signals with longer on times.

\section{REFERENCES}

Bray, D. A., Dirks, D. D., \& Morgan, D. E. Perstimulatory loudness adaptation. Journal of the Acoustical Society of America, 1973, 53, 1544-1548.

Cornsweet, T. N. The staircase method in psychophysics. American Journal of Psychology, 1962, 75, 485-491.

Derbyshire, A. J., \& Davis, H. The action potential of the auditory nerve. American Journal of Physiology, 1935, 113. 476-504.

Fraser. W. D., Petty, J. W., \& Elliott, D. N. Adaptation: Central or peripheral? Journal of the Acoustical Society of America, 1970, 47, 1016-1021.

Hattler, K. W. The type V Békésy pattern: The effects of loudness memory. Journal of Speech and Hearing Research, $1968,11,568-575$.

Mirabella, A., Taub, H., \& Teichner, W. H. Adaptation of loudness to monaural stimulationn. Journal of General Psychology, 1967, 76, 251-273.

Morgan, D. E., \& Dirks, D. D. Suprathreshold loudness adaptation. Joumal of the Acoustical Society of America, $1973,53,1560-1564$.

Petty, J. W., Fraser, W. D., \& Elliott, D. N. Adaptation and loudness decrement: $A$ reconsideration. Journal of the Acoustical Society of America, 1970, 47, 1074-1082.
SMAle, A. M., JR. Auditory adaptation. In J. Jerger (Ed.), Modern developments in audiology (1st ed.). New York: Academic Press. 1963.

Stokinger, T. E., Cooper, W. A., \& Jones, K. O. Loudness adaptation: Use of the double staircase method and heterophonic balancès. Journal of the Acoustical Society of America, 1971, 50. 144 (A).

Stokinger, T. E., Cooper, W. A., \& Meissner, W. A. Influence of binaural interaction on the measurement of perstimulatory loudness adaptation. Journal of the Acoustical Society of America, 1972, 51, 602-607. (a)

Stokinger, T. E., Cooper, W. A., \& Meissner, W. A. Loudness tracking and the staircase method in the measurement of adaptation. A udiology, 1972, 11, 161-168. (b)

Stokinger, T. E., Cooper, W. A., Meissner, W. A., \& Jones, $K$. O. Intensity, frequency, and duration effects in the measure. ment of monaural perstimulatory loudness adaptation. Journal of the Acoustical Society of A merica, 1972, 51, 608-616.

Stokinger, T. E.. \& STUdebaker, G. A. Measurement of perstimulatory loudness adaptation. Journal of the Acoustical Society of America. 1968, 44, 250-256.

WARD, W. D. Adaptation and fatigue. In J. Jerger (Ed.), Modern developments in audiology (2nd ed.). New York: Academic Press, 1973.

Wiley, T. L., Small, A. M., JR., \& Lilly, D. J. Monaural loudness adaptation. Journal of the Acoustical Society of America, 1973, 53, 1051-1054.

Zwislocki, J. J. Temporal summation of loudness: An analysis. Journal of the Acoustical Society of America, 1969, 46, 431-441.

(Received for publication December 27, 1974; revision accepted June 27,1975 .) 